\title{
Prevalence of virulence factors and antifungal susceptibility testing of Candida species isolated from clinical specimens
}

\author{
Selvaraj Stephen ${ }^{1}$, Pramodhinii S.,"*, Shanmugam J. ${ }^{3}$, Bhuvaneshwar D. ${ }^{4}$ \\ ${ }^{1}$ Professor, ${ }^{2}$ Associate Professor, ${ }^{3}$ Emeritus Professor, ${ }^{4}$ M.Sc Student, Dept. of Microbiology, Mahatama Gandhi Medical College \\ and Research Institute, Shree Balaji Vidhyapeeth (Deemed University), Puducherry, India
}

*Corresponding Author:

Email: pramodhinis@gmail.com

\begin{abstract}
Introduction: The main aim of our study is to isolate and identify Candida species from clinical samples and to detect the virulence markers and also to determine their susceptibilities to various antifungal agents.

Materials and Methods: Eighty Candida isolates from respiratory samples were included in the study. Detection of various virulence markers of candida spp such as Phospholipase, Proteinase, Hemolysin and Biofilm production was carried out by Phenotypic methods. Antifungal susceptibility testing of the isolated yeasts for Amphotericin B, Itraconazole, Fluconazole, Caspofungin and Voriconazole was determined by E-test methods.

Results: In our study, most common spp isolated was Candida albicans $(65 \%)$ followed by Candida tropicalis $(15 \%)$ and Candida glabrata(10\%).Prevalence percentage of virulence factors such as Hemolysin, Proteinase, Biofilm and Phospholipase production in Candida spp were $85 \%, 77.5 \%, 68.8 \%$ and $45 \%$ respectively. The susceptibility pattern of Candida isolates to antifungal agents showed highest resistant rate of $33.8 \%$ for Itraconazole and lowest resistant rate of $2.5 \%$ for Caspofungin.

Conclusion: Presence of virulence factors in Candida species might indicate invasiveness and its relation with infection. Hence the significance of virulence determination and antifungal susceptibility testing should be adopted as a routine procedure in the laboratory.
\end{abstract}

Keywords: Candida species, E-test, Hemolysin Biofilm formation, Proteinase, phospholipase activity.

\section{Introduction}

Most of the humans are colonized with Candida species and the leading one being C.albicans. Candidiasis is mainly an opportunistic infections affecting mucous membranes. Invasive Candidiasis can be very serious causing high mortality. Due to various risk factors, some adults develop Candidiasis and women are highly susceptible to genitourinary candidiasis as many of them carry Candida species in their vaginal tract. The new borns can develope oral Candidiasis or vulvo-vaginitis. Rarely Candidemia can progress to meningitis, leading to complications and death. ${ }^{1,2}$ The incidences of Candidiasis is on increase due to various risk factors like continuous catheterization, other prolonged invasive procedures, HIV infections, immunosuppressive therapy and other immunocompromised status. The aim of the study is to detect virulence factors and to determine the antifungal susceptibility pattern of isolated candida spp from clinical samples.

\section{Materials and Methods}

A prospective study done in a tertiary care hospital included a total of 80 Candida species isolated from respiratory samples. All suspected yeast colonies were confirmed by Gram staining and further speciated by following test like germ tube test (GTT), microscopic morphological features on Nutrient deficient medium like cornmeal agar (CMA), sugar assimilation and fermentation test.

Further speciation of Candida isolates was done by sub-culturing them on chromogenic medium (HiCHROM Candida; HiMedia, Mumbai, India) and incubated at $37^{\circ} \mathrm{C}$ for $48 \mathrm{~h}$. Presumptive identification of Candida species was done by noting the color of the colonies as per the manufacturer's Instructions in HiCHROM medium (C. albicans-green, Candida tropicalis - blue, C. krusei - pink colonies with matt surface, C. parapsilosis - cream to pale pink). (Fig. 1)

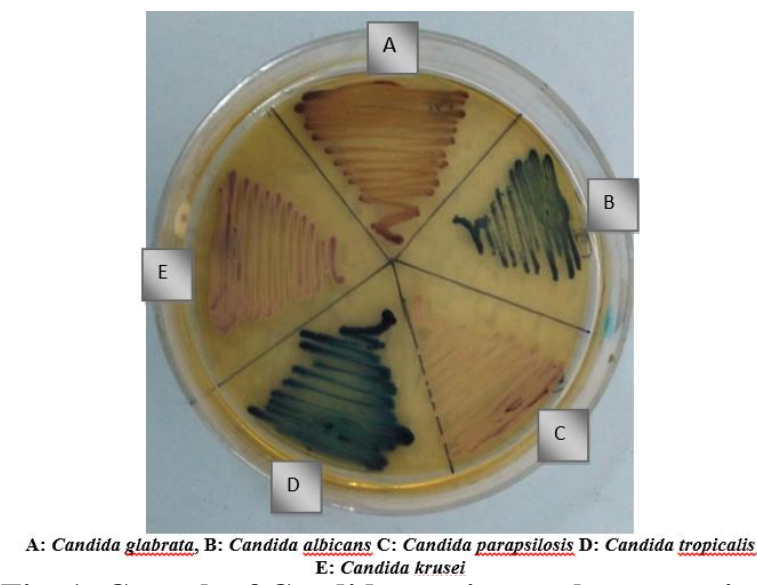

Fig. 1: Growth of Candida species on chromogenic medium 


\section{Detection of Virulence factor of candida}

The virulence factors such as Phospholipase, Proteinase,Hemolysin, and Biofilm production were detected in vitro by the following methods.

\section{Detection of Biofilm production}

Biofilm formation was detected by method proposed by Branchini et al. ${ }^{3}$ A loopful of colony from SDA plate was inoculated into $10 \mathrm{ml}$ of Sabouraud's liquid broth with $8 \%$ glucose supplemented. The tubes were further incubated at $37^{\circ} \mathrm{C}$ for $24 \mathrm{hrs}$. Following which the broth was aspirated from tube and walls were stained using safranin stain. Biofilm formation was graded as negative $(0+)$ and $1+, 2+$ and $3+$ means weak,moderate and strong positivity.

2. Proteinase detection

The proteinase activity was detected by the slightly modified Staib et al, method. ${ }^{4}$ The medium used was Bovine serum albumin medium. On the surface of the medium the wells were punched out and yeast suspension (approximately $10^{8}$ yeast cells $/ \mathrm{ml}$ ) was inoculated into it. The plates were incubated for $48 \mathrm{hrs}$ at $37^{\circ} \mathrm{C}$. After incubation, the plates were fixed using $20 \%$ trichloracetic acid following which staining done by $1.25 \%$ amidoblack. Further decolourisation of the stained plates were done by $15 \%$ acetic acid. The diameter of unstained area surrounding the well is measured. The proteinase activity $(\mathrm{Pz})$ is the ratio of the diameter of the well to the diameter of the proteolytic unstained zone. If the value of $\mathrm{Pz}=1$, indicates absence of proteinase activity and low $\mathrm{Pz}$ means high proteinase enzyme activity.

3. Detection of Phospholipase activity

Phospholipase activity of Candida species was detected by modified method of Samaranayake $e t$ $a l .{ }^{5}$ The medium used is egg yolk agar medim. The wells were punched out on the surface of the well and $10 \mu \mathrm{l}$ aliquots of the yeast suspension was inoculated in the corresponding wells. The plates were further incubated for $48 \mathrm{hrs}$ at $37^{\circ} \mathrm{C}$ for. The phospholipase activity (Pz value) is the measure of ratio of the diameter of the well to the diameter of the precipitation zone around the well. The value of $\mathrm{Pz}=1$, means phospholipase activity is absent and low value of $\mathrm{Pz}$ means more production of phospholipase enzyme. (Fig. 2)

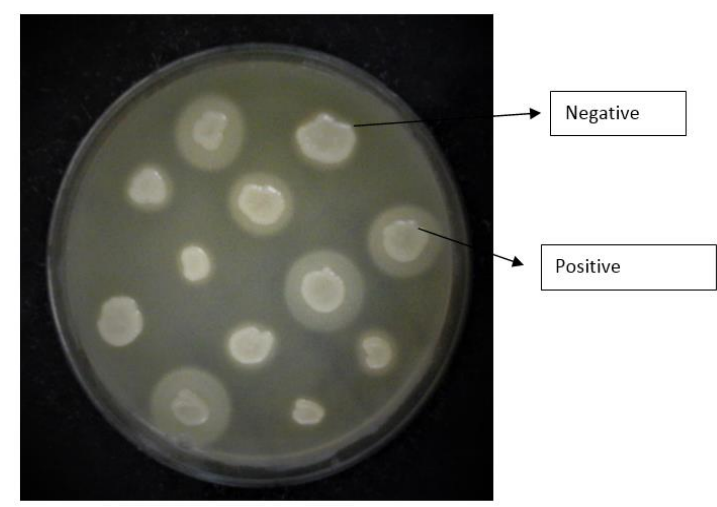

Fig. 2 Phospholipase production of Candida sppon egg yolk agar

4. Detection of Haemolysin activity

It was detected by inoculating the colony in SGA blood plate with $3 \%$ glucose added. The plate was further more incubated for 2 days at 37 degree $\mathrm{C}$ in $5 \% \mathrm{CO}$. Haemolytic index ( $\mathrm{Hz}$ value) is measure of ratio of the diameter of the colony to that of diameter of translucent zone of haemolysis (in $\mathrm{mm}) ., 6$

\section{Antifungal susceptibility testing}

Antifungal susceptibility testing of the isolated yeasts was done by E-test methods (Fig. 3) for Amphotericin B, Itraconazole, Fluconazole, Caspofungin and Voriconazole. Interpretation of susceptibility was performed by applying the breakpoints criteria defined by the CLSI in $2012^{[8]}$ as well as EUCAST. ${ }^{9}$

The MIC range of drugs used in this study were Amphotericin B (0.002 to $32 \mu \mathrm{g} / \mathrm{ml})$, Fluconazole (0.016 to $256 \mu \mathrm{g} / \mathrm{ml})$, Voriconazole (0.002 to 32 $\mu \mathrm{g} / \mathrm{ml})$, Itraconazole $(0.002$ to $32 \mu \mathrm{g} / \mathrm{ml})$ and Caspofungin (0.002 to $32 \mu \mathrm{g} / \mathrm{ml})$.

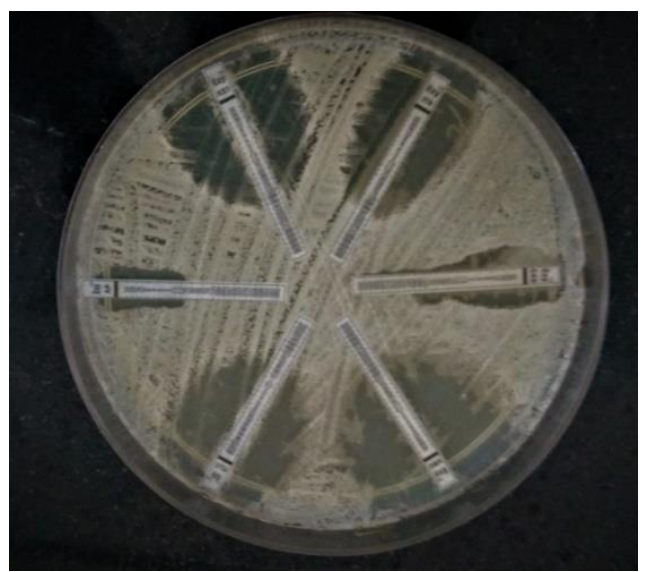

Fig. 3: Antifungal susceptibility testing of Candida spp by E-test method 


\section{Results}

In the current study, the most common candida species isolated was Candida albicans $(65 \%)$, which was followed by $C$. tropicalis $12(15 \%)$, C. glabrata 8(10\%), C.parapsilosis 4(5\%) and C. krusei 4(5\%) (Table 1)

Detection and prevalence of virulence factors in Candida spp showed $85 \%$ of isolates were positive for Hemolysin and $77.5 \%$ for Proteinase followed by positivity of $68.8 \%$ for Biofilm and $45 \%$ for Phospholipase. (Table 2)

Testing for antifungal susceptibility pattern showed resistant rate of $33.8 \%(27 / 80)$ for Itraconazole, $13.7 \%$ $(11 / 80)$ for AmphotericinB, $5 \%(4 / 80)$ for Fluconazole \& Voriconazole and 2.5\% (2/80) for Caspofungin. (Table 3)

Table 1: Percentage of Candida species isolated from clinical samples

\begin{tabular}{|l|c|}
\hline \multicolumn{1}{|c|}{ Candida species } & No (\%) \\
\hline C. albicans & $52(65 \%)$ \\
\hline C. tropicalis & $12(15 \%)$ \\
\hline C. glabrata & $8(10 \%)$ \\
\hline C.parapsilosis & $4(5 \%)$ \\
\hline C. krusei & $4(5 \%)$ \\
\hline Total & 80 \\
\hline
\end{tabular}

Table 2: Prevalence of virulence factors in Candida spp

\begin{tabular}{|l|c|c|}
\hline Virulence factors & Positive & Negative \\
\hline Biofilm & $55(68.8 \%)$ & $25(31.2 \%)$ \\
\hline Hemolysin & $68(85 \%)$ & $12(15 \%)$ \\
\hline Phospholipase & $36(45 \%)$ & $44(55 \%)$ \\
\hline Proteinase & $62(77.5 \%)$ & $18(22.5 \%)$ \\
\hline
\end{tabular}

Table 3: Antifungal susceptibility pattern of Candida spp.

\begin{tabular}{|l|c|c|}
\hline $\begin{array}{l}\text { Antifungal } \\
\text { agents }\end{array}$ & Sensitive & Resistant \\
\hline Amphotericin B & $69(86.2 \%)$ & $11(13.7 \%)$ \\
\hline Itraconazole & $53(66.2 \%)$ & $27(33.8 \%)$ \\
\hline Fluconazole & $76(95 \%)$ & $4(5 \%)$ \\
\hline Caspofungin & $78(97.5 \%)$ & $2(2.5 \%)$ \\
\hline Voriconazole & $76(95 \%)$ & $4(5 \%)$ \\
\hline
\end{tabular}

\section{Discussion}

Though more than 80 Candida species are described, only about 12-15 species can cause diseases in humans and most common is C.albicans followed by C.tropicalis, C.glabrata, C.krusei, C.kefir, C.guilliermondii, C.parapsilosis and recently C.dubliniensis. Candida albicans and other species can cause vulvo-vaginitis, oral candidiasis, UTI, esophagitis (mostly among HIV patients), meningitis, Candidemia and other opportunistic infections The risk factors for the development of Candida infections and various types of disease are: Prolonged antibiotic therapy, HIV infection and other Immuncompromised status, prolonged catheterization, various other invasive procedures, artiificial organs and other plastic/metal implants

In our study, the most predominant isolate of candida species was Candida albicans $(65 \%)$, which is followed by non albicans Candida spp. which accounts for $35 \%$. This is well comparable to previous other studies where among all candida spp, C. albicans was the most predominant isolate from the clinical samples. ${ }^{10,11}$

But there are few other studies, which have proven that non albicans Candida species is emerging and showing increased prevalence. ${ }^{12,13}$ There are many types of virulence factors described for Candidiasis. The leading ones are slime production and adhesion factors. The others being hemolysisn, phospholipase and proteinases.

The present study done on detection and prevalence of virulence factors in Candida spp showed $85 \%$ of isolates were positive for Hemolysin and $77.5 \%$ for Proteinase followed by positivity of $68.8 \%$ for Biofilm and $45 \%$ for Phospholipase. A similar study conducted by Deepa et al ${ }^{14}$ showed that the prevalence of virulence factors in Candida spp such as hemolysin, proteinase, biofilm \& phospholipase were 63\%, $86.8 \%, 78.9 \%$ and $52.6 \%$ respectively much comparable to our study.

In our study, haemolysin was the major virulence factor expressed by Candida isolates $85 \%$ irrespective of its species in comparable to study by Ghosh et al. ${ }^{15}$ Several other studies have shown $60.9 \%, 52.6 \%, 33 \%$ positivity of phospholipase activity. ${ }^{10,14,16}$ In our study we have recorded $45 \%$ phospholipase activity by Candida spp.

In current study $77.5 \%$ of Candida spp were positive for Proteinase production. In concurrence with this report, $75 \%, 86.8 \%$ percentage of protease enzyme production have been reported in other studies. ${ }^{10,14} \mathrm{In}$ the present study $68.8 \%$ of Candida isolates were biofilm producers. Study by Jose et al., have reported similar percentage. ${ }^{10}$

Due to rapid advancement in complicated surgeries and many invasive procedures, Candida species invade blood stream and later affect many organs of the body. Candida species which develop drug resistance in due course invade bloodstream can lead to high mortality upto $35 \%$. During the past two decades, the invasive Candida infections increased by about $20 \%$.

E-test strips are commercially available for amphotericin $\mathrm{B}$, fluconazole itraconazole, flucytosine, voriconazole, posaconazole, and caspofungin. One of the most remarkable advantage of E-test method is its user-friendly. In addition, irrespective of the test medium used, E-test has once been found to be most reliable and effective method when compared to other 
method like reference microdilution method for detection of resistance to amphotericin B in Candida. ${ }^{17}$

In the present study on antifungal susceptibility pattern of Candida spp showed high resistant rate of $33.8 \%$ for Itraconazole followed by Amphotericin B $(13.7 \%)$. Among 80 Candida isolates, resistant rate of $5 \%$ for Fluconazole \& Voriconazole followed by $2.5 \%$ for Caspofungin.were recorded. In this study we found that Caspofungin, fluconazole and voriconazole continue to be active against most isolates of Candida. We have also observed that resistance to fluconazole predicted resistance to voriconazole well agreed with study by Lyon $e t$ al. ${ }^{18}$

Detection of virulence factors like haemolysin proteinase, phospholipase, and biofilm production will help us to understand the relationship between the infection and Candida species isolated. In recent years, a gradual increase in the fungal diseases and the widespread use of empirical antifungals caused the emergence of resistant strains of fungi. Thereby, the need for in vitro antifungal susceptibility testing are increasing nowadays to select appropriate and effective antifungal therapy. Treatment should be started only to those who are having clinical symptoms and after confirming with laboratory diagnosis. Hence, the significance of virulence determination in rational antifungal therapy cannot be ignored and should be adopted as a routine procedure in the laboratory.

\section{References}

1. Farrugia MK, Fogha EP, Miah AR, Yednock J, CarlPalmer H, Guilfoose J. Candida meningitis in an immunocompetent patient detected through $(1 \rightarrow 3)$-betad-glucan. Int. J Infect Dis. 2016; 51:p 25-6.

2. Voice RA1, Bradley SF, Sangeorzan JA, Kauffman CA. Chronic candidal meningitis: an uncommon manifestation of candidiasis. Clin Infect Dis. 1994 Jul;19(1):p 60-6.

3. Branchini ML, Pfaller MA, Rhine- chalk berg J, Frempong T, Isenberg HD. Genotype variation and slime production among blood and catheter isolates of $C$ parapsilosis. J Clin Microbiol. 1994;32:452-6.

4. Staib F. Serum-proteins as nitrogen source for yeast like fungi. Sabouraudia. 1965;4:187-93.

5. Samaranayake YH, Dassanayake RS, Jayatilake JA, Cheung BP, Yau JY, Yeung KW, et al. Phospholipase B enzyme expression is not associated with other virulence attributes in Candida albicans isolates from patients with human immunodeficiency virus infection. J Med Microbiol. 2005;54:583-93.

6. Sacristan B, Blanco MT, Galan-Ladero MA, Blanco J, Perez-GiraldoC,Gomez-Garcia AC. Aspartyl proteinase, phospholipase, hemolytic activities and biofilm production of Candida albicans isolated from bronchial aspirates of ICU patients. Med Mycol 2011;49:94-7.

7. Shehabi AA, NazzalSA,Dajani N. Putative Virulence Factors of Candida Species Colonizing Respiratory Tracts of Patients. MicrobEcol Health Dis 2004;16:214-7.

8. Clinical and Laboratory Standards Institute. Reference Method for Broth Dilution Antifungal Susceptibility Testing of Yeast; Approved Standard-Third Edition. CLSI document M27-A3. Wayne: Clinical and Laboratory Standards Institute; 2008.
9. Arendrup MC, Cuenca-Estrella M, Lass-Flörl C, Hope W. EUCAST technical note on the EUCAST definitive document EDef 7. 2: method for the determination of broth dilution minimum inhibitory concentrations of antifungal agents for yeasts EDef 7.2 (EUCAST-AFST). Clin Microbiol Infect. 2012;18:E246-E247.

10. Jose NC, Mudhigeti N, Asir J, Chandrakesan SD. Detection of virulence factors and phenotypic characterization of Candida isolates from clinical specimens.Journal of Current Research in Scientific Medicine 2015;1(1):27-31.

11. Khan PA, Fatima N, Nabeela, Jahan S, Khan HM, Malik A.Antifungal Susceptibility Pattern of Candida Isolates from a Tertiary Care Hospital of North ndia: A Five Year Study. Int. J. Curr. Microbiol. App. Sci 2015;1:177-81.

12. Manchanda, V., Agarwal, S., Verma, N.2011. Yeast identification in routine clinical microbiology laboratory and its clinical relevance. Indian J. Med.

Microbiol.,29(2):172.

13. Ragini A.K., SandhyaBelawadi, GaytriDevi, Indumal. 2001. Characterisation and antifungal susceptibility testing for Candida in a tertiary care hospital. J. Health Sci. Res., 2(2):1-12.

14. Deepa K, Jeevitha T, Michael A. Invitro evaluation of virulence factors of candida spcies isolated from oral cavity. J Microbiol. Antimicrob 2015;7(3):28-32.

15. Ghosh RR, Ghosh M, Chatterjee M, Banerjee M. In vitro demonstration of potential virulence determinants among clinical isolates of various Candida species and its clinical implication in a Teaching Hospital in Eastern India. Indian J. Med. Microbiol. 2016; 34( 23):406-7.

16. Sachin C.D, Ruchi K, Santosh S. In vitro evaluation of proteinase, phospholipase and haemolysin activities of Candida species isolated from clinical specimens. Int J Med Biomed Res 2012;1(2):153-7.

17. Peyron F, Favel A, Michel-Nguyen A, et al. Improved detection of amphotericin B-resistant isolates of Candida lusitaniae by Etest. J Clin Microbiol 2001; 39: 339-42.

18. Lyon GM, Karatela S, Sunay S, Adiri Y. Antifungal Susceptibility Testing of Candida Isolates from the Candida Surveillance Study. J Clin Microbiol 2010:48(4);1270-5.

How to cite this article: Stephen S, Pramodhinii S, Shanmugam J, Bhuvaneshwar D. Prevalence of virulence factors and antifungal susceptibility testing of Candida species isolated from clinical specimens. Indian $\mathbf{J}$ Microbiol Res 2018;5(1):106-109. 